Communications in Physics, Vol. 24, No. 3 (2014), pp. 207-215

DOI:10.15625/0868-3166/24/3/4070

\title{
SIZE DEPENDENT MELTING OF SILICON NANOPARTICLES
}

\author{
NGUYEN THI THUY HANG \\ Department of Applied Physics, Ho Chi Minh University of Technology, Vietnam National \\ University of Ho Chi Minh City \\ E-mail: hangthuynguyen2001@yahoo.com \\ Received 30 May 2014 \\ Accepted for publication 21 August 2014
}

\begin{abstract}
Melting of crystalline silicon nanoparticles is studied by molecular dynamics (MD) simulations using StillingerWeber potential. Models are heated up from a crystalline to a normal liquid state. Temperature dependence of total energy and the Lindemann ratio exhibit a first-order-like behavior of the transition at the melting point. Heat capacity of the system presents a single peak at around the melting point. The size dependent melting is presented. As the size of the nanoparticles increases, the variation of the melting point becomes more monotonic and the temperature range of bistability shifts to higher temperatures. In large nanoparticles, the proportion of interior atoms increases and the average potential energy per atom converges to the bulk or thin films.
\end{abstract}

Keywords: thermodynamics of melting, molecular dynamics simulations, size dependent melting, silicon nanoparticles.

\section{INTRODUCTION}

Melting of crystalline nanoparticles has been under much attention for a long time due to the scientific and technological importance of the phenomenon [1]. Since Takagi first reported the size dependent melting temperature of small particles by means of transmission electron microscope [2], researchers have paid more attention to this basic but still unclear phenomenon [3-6]. Now it is found that the melting temperature of metallic [3,4], organic [6] and semiconductors [5] nanoparticles decreases with decreasing their particle size. In particular, silicon nanoparticles are interesting because the poor optical characteristics of silicon due to its indirect band structure are drastically improved by reducing the particle size [7].

It is well known that melting point depression is due to the large influence of the surface energy on the properties of small particles [8]. Such energy are generally negligible in the bulk, but at the nanoscale, suface-to-volume ratios become very larges. Several potentials including twoand three-body terms have been used to compute the structural energies of covalent crystals such as silicon [11-13]. However, for silicon as well as the covalent materials, pair potentials alone are insufficient to describe the equilibrium diamond lattice. In particular, the Stillinger-Weber (SW) potential [14] has nine parameters that were adjusted to fit the silicon properties of condensed phases such as bond length, cohesive energy, melting temperature to satisfy qualitatively 
the Lindemann melting criterion for solids and to produce the property of shrinking when silicon melts.

There are numbers of studies focusing on silicon using SW and other potentials [15-25]. While experiments mainly focus on studying zone melting and the mechanism of formation of grains using laser energy $[15,17-21]$, some simulations such as study the velocity of melting on the (110) silicon with free surface [16], Zhang et al. focus on the volume change from solid to liquid and the curvature of the interface between the solid and the liquid phases [24]. It is well known that, in a macroscopic sample, the surfaces and interfaces are usually proportionately so small that they have only a small effect on the overall properties of a material. At the nanoscale, however, they can be a large fraction of the material sample itself and strongly effect on the thermodynamic properties and structures of material. It motivates us to present a whole picture about the size dependent melting of silicon nanoparticles.

The melting transitions of nanoparticles have been characterized by calculating various dynamic or structural properties at different temperatures. The properties used to characterize melting include the Lindemann ratio [26-32], radial distribution functions, and many different types of energy-temperature or heat capacity-temperature plots [33-37]. Details about the calculations can be seen in Sec. II. Results and discussions about the size dependent melting of silicon nanoparticles can be found in Sec. III. Conclusions are given in the last section of the paper.

\section{CAlCulation}

The initial object is diamond cubic structure of crystalline silicon. We use SW potential for describing the interaction between atoms in the models. The SW potential is given below [14]:

$$
\begin{gathered}
E=\sum_{i} \sum_{j>i} \phi_{2}\left(r_{i j}\right)+\sum_{i} \sum_{j \neq i} \sum_{k \neq j} \phi_{3}\left(r_{i j}, r_{i k}, \theta_{i j k}\right), \\
\phi_{2}\left(r_{i j}\right)=A_{i j} \varepsilon_{i j}\left[B_{i j}\left(\frac{\sigma_{i j k}}{r_{i j}}\right)^{p_{i j}}-\left(\frac{\sigma_{i j k}}{r_{i j}}\right)^{q_{i j}}\right] \exp \left(\frac{\gamma_{i k} \sigma_{i k}}{r_{i k} a_{i k} \sigma_{i k}}\right), \\
\phi_{3}\left(r_{i j}, r_{i k}, \theta_{i j k}\right)=\lambda_{i j k} \varepsilon_{i j k}\left[\cos \theta_{i j k}-\cos \theta_{0 i j k}\right]^{2} \exp \left(\frac{\gamma_{i j} \sigma_{i j}}{r_{i j} a_{i j} \sigma_{i j}}\right) \exp \left(\frac{\gamma_{i k} \sigma_{i k}}{r_{i k} a_{i k} \sigma_{i k}}\right),
\end{gathered}
$$

where $\phi_{2}\left(r_{i j}\right)$ and $\phi_{3}\left(r_{i j}, r_{i k}, \theta_{i j k}\right)$ are two-body and three-body term, respectively; $r_{i j}$ the distance between the atoms $i$ and $j ; \theta_{i j k}$ the angle between the vectors $r_{i j}$ and $r_{i k} ; A_{i j}, B_{i j}, p_{i j}, q_{i j}, \lambda_{i j k}, \gamma_{i j}$ and $a$ are the dimensionless parameters, the parameter has a dimension of energy, the parameter has a dimension of length. Parameters were chosen based upon the properties of crystalline and liquid silicon.

To perform the calculations we use the software package LAMMPS (Large-Scale Atomic/Molecular Massively Parallel Simulator), designed to solve various problems by the methods of classical molecular dynamics [38].

In present study, models with 10557, 20521 and 32768 atoms are presented and the simulation scenario of each model includes 3 stages as follows.

Stage 1: The initial crystalline silicon models containing desired identical atoms of $\mathrm{Si}$ in a sphere at the density $\rho=2.3290 \mathrm{~g} / \mathrm{cm}^{3}$ under periodic boundary conditions (PBCs) have been relaxed in NVT ensemble for $10^{5} \mathrm{MD}$ steps at $T_{0}=50 \mathrm{~K}$. Note that $1 \mathrm{MD}$ step takes 0.001 picoseconds. 
Stage 2: After that all periodic boundaries are relaxed by open boundary conditions with an elastic reflection behavior. The system is left to equilibrate further for $2 \times 10^{5} \mathrm{MD}$ steps at $T_{0}=50 \mathrm{~K}$ using NVT ensemble simulation corresponding to the new boundaries of the simulation cell.

Stage 3: A temperature $T_{i}\left(T_{i}=2500 \mathrm{~K}\right)$ is chosen. It is higher than the melting point of $\mathrm{Si}$ to guarantee that at temperature $T_{i}$ all atoms become liquid. The system is heated from temperature $T_{0}$ to $T_{i}$ in the NoseHoover canonical (NVT ensemble). The heating rate is $1.225 \times 10^{11} \mathrm{~K} / \mathrm{s}$.

\section{RESULTS AND DISCUSSIONS}

Temperature dependence of potential energy per atom of the system is interesting because we can detect various phenomena including phase transitions from the curve. In the low temperature region, total energy linearly increases with temperature since the system remains in solid state and vibrational motion of atoms around their equilibrium positions dominates in the system. Starting of potential energy to deviate from a linearity of a low temperature region at around $T_{x}=1608 \mathrm{~K}$ is due to the occurrence of significant amount of the liquidlike atoms in the surface shell of nanoparticles, i.e. contribution of an anharmonic motion of atoms is significant.

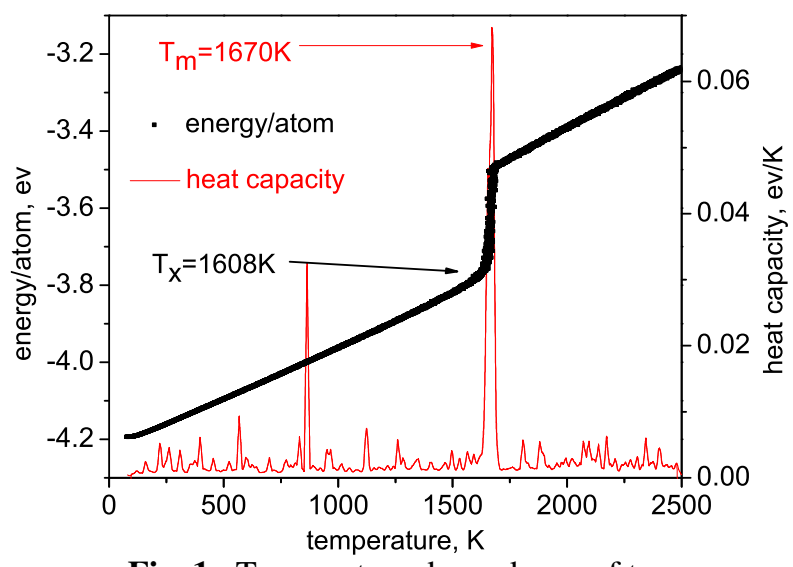

Fig. 1. Temperature dependence of total energy per atom and heat capacity. $T_{x}=1608 \mathrm{~K}$ can be assumed as the limit of thermal stability of crystalline matrix since at which significant amount of the liquidlike atoms occurs and destruction of crystalline matrix is started. This temperature is similar to the point of the onset of surface melting (denoted as TW) proposed in Ref. [1], or $T_{s t}$ the point at which particles begin to melt at the surface of $\mathrm{Pb}$ nanoparticles as denoted in reference [39]. Potential energy intensively increases upon further heating and reaches a next linear part of the high temperature region which is related to the melted stage of the system (see Fig. 1). The heat capacity calculated via the simple relation: $C_{p}=\frac{\Delta E}{\Delta T}$. Here, $\Delta E$ is a discrepancy in total energy per atom upon heating from $T_{1}$ to $T_{2}$ with $\Delta T=T_{1}-T_{2}=12 \mathrm{~K}$. One can see that heat capacity exhibits a sharp peak at around $T_{m}=1670 \mathrm{~K}$ which is the melting point of nanoparticles. The left bound of the peak of the heat capacity, i.e. $T_{x}=1608 \mathrm{~K}$, can be considered as a lower bound of the melting region at which a large amount of liquidlike atoms occurs and their number strongly increases with temperature, i.e. a massive collapse of crystalline lattice is started. A sharp peak of the heat capacity exhibits a first-order like behavior of the melting of nanoparticles. However, due to surface effects melting of crystalline nanoparticles does not occur at a certain point, i.e. melting proceeds over a finite temperature range like that found and discussed for melting of various metal nanoparticles such as $\mathrm{Al}, \mathrm{Ni}$, etc. [40-42] and the melting point is a bit lower than experimental result $1685 \mathrm{~K}$ [43].

We present the size dependent melting via temperature dependence of total energy per atom of different sizes of nanoparticles: 10557, 20521 and 32768 atoms (see Fig. 2a). Melting point 


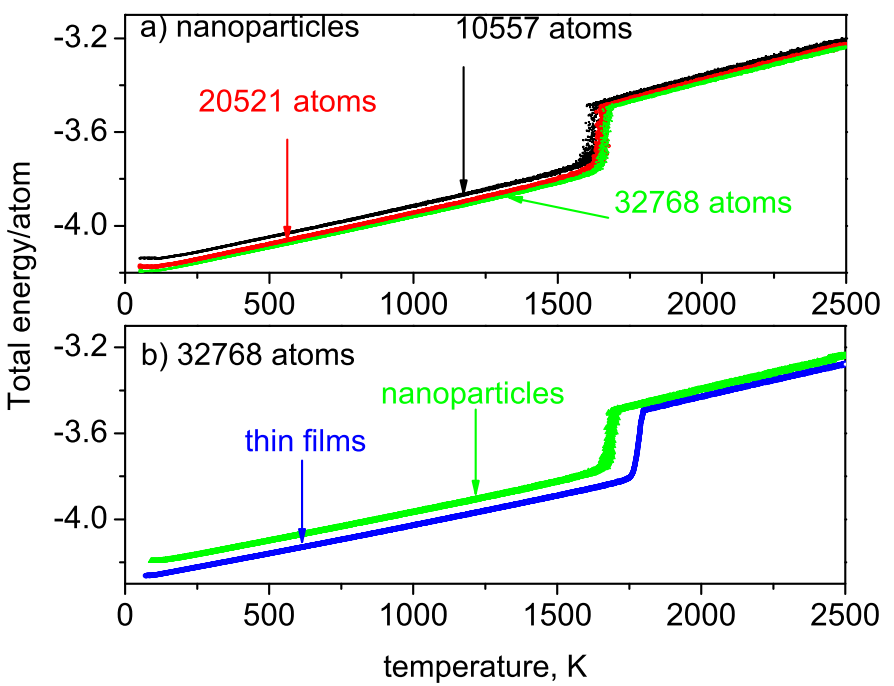

Fig. 2. Temperature dependence of total energy per atom for different sizes of model: a) 10557 atoms-black color, 20521 atoms-red color, 32768 atoms-green color. b) models with 32768 atoms: nanoparticles-green color, thin films-blue color.

of model with 32768 atoms (green color) is about $1670 \mathrm{~K}$ (very close to the experimental one $1685 \mathrm{~K}$ ). But when the number of atoms is decreased to 20521 (red color) and 10557 atoms (black color), melting point is about $1650 \mathrm{~K}$ and $1615 \mathrm{~K}$, respectively. We also present the temperature dependence of total energy per atom of nanoparticles and thin films of models with the same number of atoms (32768 atoms) see Fig. 2b. It clearly shows that melting point of nanoparticles $(1670 \mathrm{~K})$ is lower than thin films $(1779 \mathrm{~K})$ due to the high fraction of under-coordinated surface atoms of nanoparticles. One can see that in large nanoparticles, the proportion of interior atoms increases and the average potential energy per atom converges to the bulk or thin films.

The trajectories of total potential energy per atoms in Figs. 1,2a have the same behavior i.e. the potential energy of trajectory jumps between low values, corresponding to the solid state of nanoparticles, and high values, which correspond to the liquid state; that is, the particle undergoes dynamic coexistence melting and we will call this temperature range as bistable. As the temperature of the trajectories is increased, the probability of the nanoparticle residing in the solid state decreases, and finally a temperature is reached at which the nanoparticle only is stable in the liquid state. It is seen in Fig.2a that the range of bistability shifts to higher temperatures as the size of the nanoparticle increases.

We also calculate important quantity, the Lindemann ratio, which is given for ith atom [4143]: $\delta_{i}=\left\langle\Delta r_{i}^{2}\right\rangle^{\frac{1}{2}} / \bar{R}$. Here, $\Delta r_{i}^{2}$ is the mean-squared displacement (MSD) of the ith atom and $\bar{R}=2.33$ Ais a nearest neighbor distance in our diamond cubic structure of crystalline silicon at low temperature. In the atomic motions, there are two kinds of contributions to the MSD: the first one is vibrational motion and the second one is diffusive displacement. The diffusive motions (liquidlike motion [44]) are thought to be related to the local instability in the system, which play the key-role of the melting mechanism [44,45]. Therefore, the best relaxation time for identifying is proposed to be not larger than several picoseconds [46]. In present work, the value for $\delta_{L}$ is 
calculated via relaxation of model for $5000 \mathrm{MD}$ steps (or 5ps) at a given temperature, i.e., the time is large enough for atoms to diffuse if they are liquidlike [47]. The mean Lindemann ratio $\delta_{L}$ of the system is defined by averaging of $\delta_{i}$ overall atoms, $\delta_{L}=\sum_{i} \delta_{i} / N$. As shown in Fig. 3, the behavior of temperature dependence of $\delta_{L}$ is similar to that of total energy per atom indicating a close correlation between two quantities. At $T=T_{x}$, critical value for the Lindemann ratio is $\delta_{C}=0.19$. Critical value for $\delta_{C}$ is taken as 0.15 or from 0.05 to 0.20 of the nearest neighbor distance depending on the crystal structure, nature of the interparticle interaction and magnitude of quantum effects (see [48] and references therein). One can see that temperature dependence of total energy and the Lindemann ratio exhibit a first-order-like behavior of the transition at a melting point.

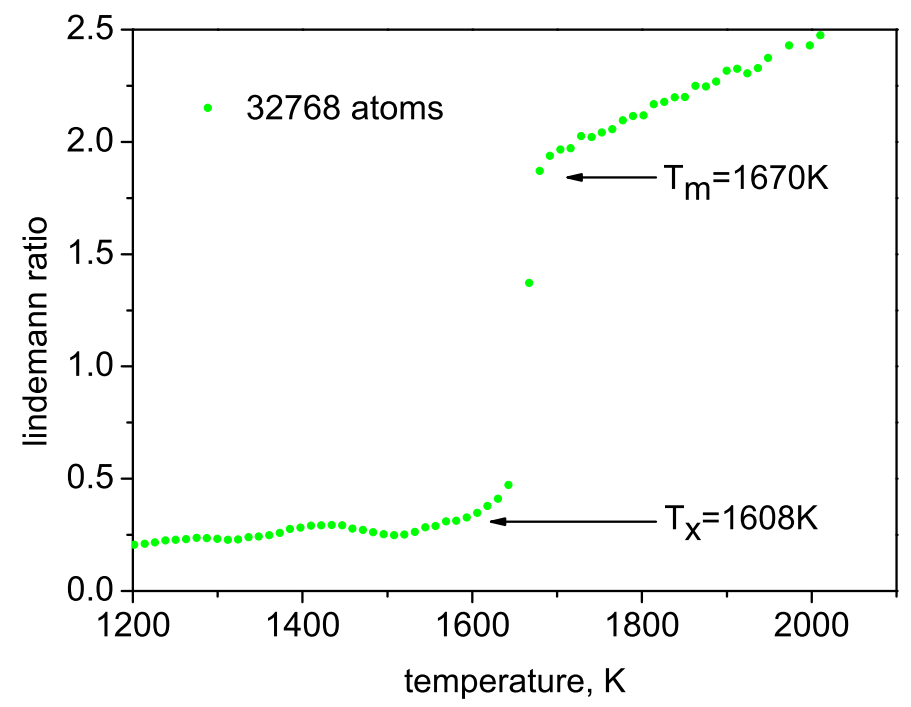

Fig. 3. Temperature dependence of the Lindemann ratio.

The other nanoparticle sizes show similar behavior (Fig. 4a). The Lindemann ratio is initially stable at a low value, up to a certain temperature, above which it starts to increase and fluctuate. After a sharp rise, the Lindemann ratio will then increase monotonically as temperature is increased. For solid nanoparticles, at low temperatures the atoms are confined to fixed sites and the Lindemann ratio converges to a value which is characteristic of bulk phases. After melting, the Lindemann ratio of the liquid nanoparticles almost have the same value. At bistable temperature range, however, the Lindemann ratio shifts to higher temperatures as the size of the nanoparticle increases (Fig. 4a). We also present the Lindemann ratio of nanoparticles and thin films of models with the same number of atoms (32768 atoms) see Fig. 4b. One can see that in large nanoparticles, the Lindemann ratio converges to the thin films.

Melting process can be monitored via analyzing spatio-temporal arrangements of liquidlike atoms occurred in the models during heating $[48,49]$. Liquidlike atoms can be detected via using the Lindemann melting criterion, i.e. atoms with $\delta_{L}<\delta_{C}$ are classified as solidlike atoms and with $\delta_{L}>\delta_{C}$ are classified as liquidlike. Note that such a method of detecting of the liquidlike/solidlike 


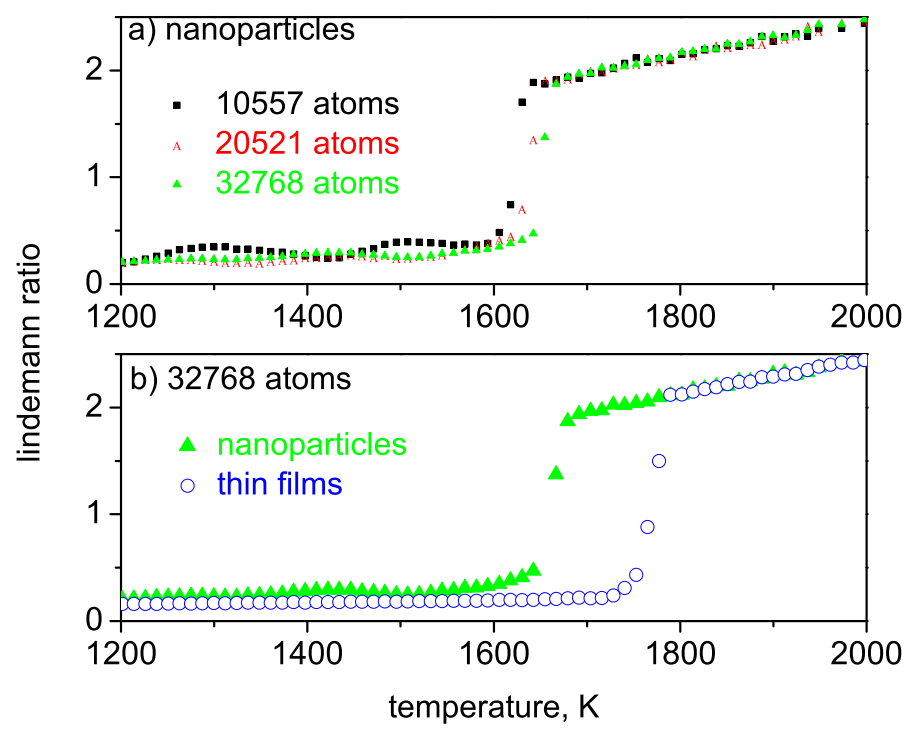

Fig. 4. Temperature dependence of the Lindemann ratio for different sizes of model: a) 10557 atoms-black color, 20521 atoms-red color, 32768 atoms-green color. b) models with 32768 atoms: nanoparticles-green color, thin films-blue color.

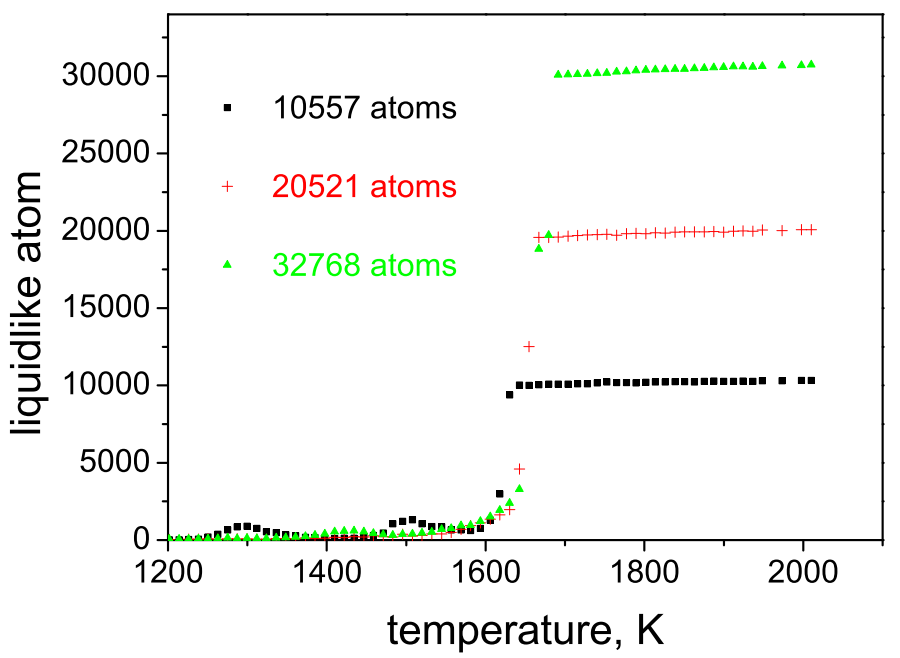

Fig. 5. Temperature dependence of the liquidlike atoms for different sizes of model: a) 10557 atoms-black color, 20521 atoms-red color, 32768 atoms-green color.

atoms has been successfully employed for studying of melting of various materials (see for example [48-50]). At temperature far below $1600 \mathrm{~K}$, atoms of three models exhibit solidlike, however, at temperature $T=1615 \mathrm{~K}, T=1630 \mathrm{~K}$ and $T=1670 \mathrm{~K}$ all atoms of models with 10557,20521 and 32768 atoms become liquidlike, respectively. One can see that temperature dependence of 
liquidlike atoms upon heating is similar to that found for the energy per atoms and the Lindemann ratio (see Figs.1-4). This indicates a close correlation between three quantities in the melting process.
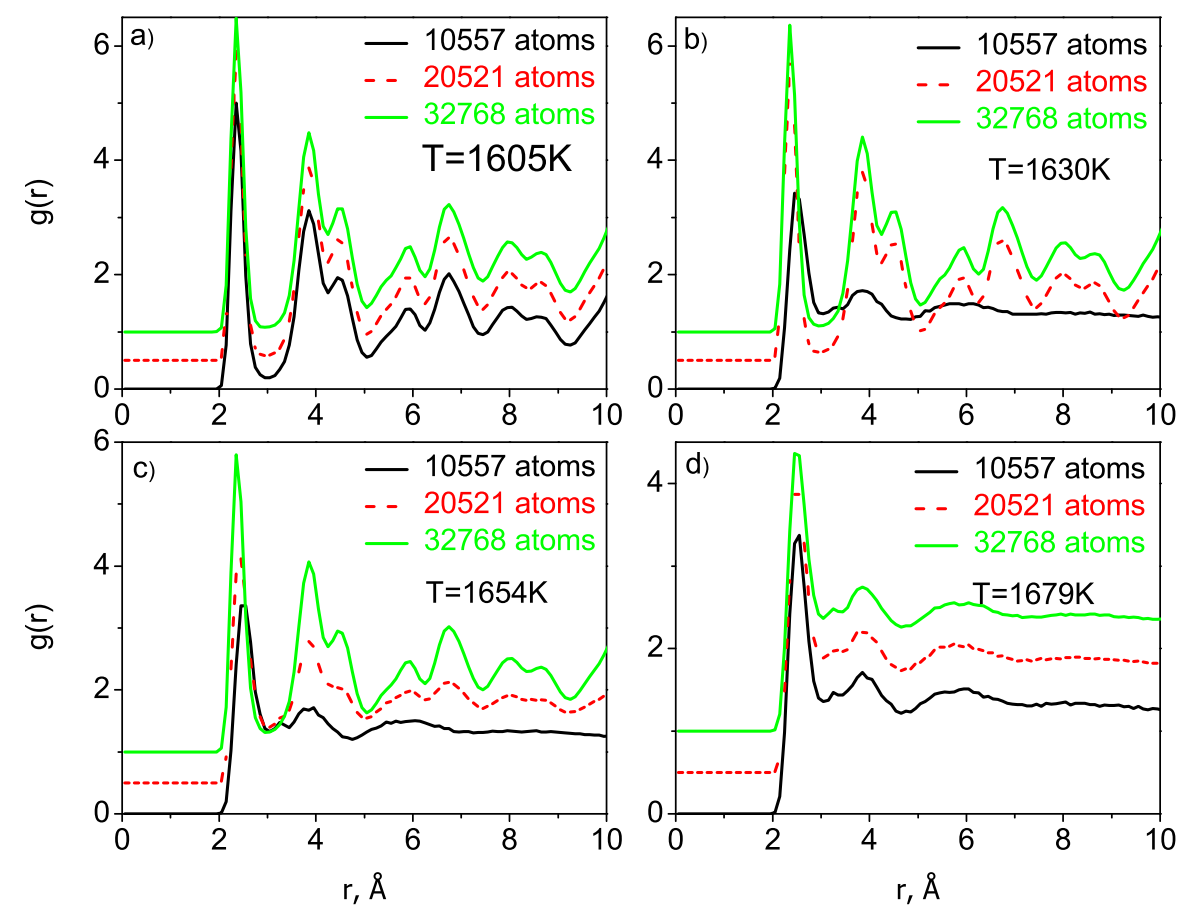

Fig. 6. Radial distribution function at different temperatures: 10557 atoms-black color, 20521 atoms-red color, 32768 atoms-green color.

On the other hand, liquidlike behavior of nanoparticles can be seen via radial distribution function (RDF) of a given model if it has no peaks related to the crystalline state of the diamond cubic structure of crystal. The transition from crystal to liquid upon heating can be seen via evolution of radial distribution function (RDF), i.e. the peaks in RDF related to the diamond cubic structure of crystal become weaker with temperature and almost disappear at around melting point. At temperature higher melting point, RDF exhibits a liquid like behavior. Temperature dependence of RDF of each model is given as followings (Fig. 6); First, at $T=1605 \mathrm{~K}$ all models of nanoparticles are till crystalline solid since RDF of each model exhibits many peaks related to the crystalline structure (Fig. 6a). Next, temperature is increased, model with 10557 atoms fully becomes liquidlike at $T=1630 \mathrm{~K}$ while models with 20521 and 32000 atoms are till crystalline solid (Fig. 6b). This absolutely corresponds with situation in Fig. 2a i.e. melting point of model with 10557 atoms is $1615 \mathrm{~K}$. Then, models with 20521 atoms becomes (Fig. 6c) at $T=1654 \mathrm{~K}$. Finally, at $T=1679 \mathrm{~K}$, all three models become liquidlike (Fig. 6d). One can see that temperature which is showed in Figs. 1,2,6 indicates a close correlation between two quantities in the melting process 


\section{CONCLUSION}

Molecular dynamics simulations of silicon nanoparticles were carried out using the SW potential. Thermodynamics and atomic mechanism of melting of silicon nanoparticles with different sizes (10557, 20521 and 32000 atoms) has been characterized by calculating the potential energy per atom, heat capacity, the Lindemann ratio and RDF. Heat capacity of the system exhibits a single peak at around the melting point.Temperature dependence of total energy and the Lindemann ratio exhibit a first-order-like behavior of the transition at a melting point. The size dependent melting is presented. As the size of the nanoparticles increases, the variation of the melting point becomes more monotonic and the temperature range of bistability shifts to higher temperatures. In large nanoparticles, the proportion of interior atoms increases and the average potential energy per atom converges to the bulk or thin films.

\section{ACKNOWLEDGMENT}

Authors thank for financial support from the National Foundation for Science and Technology Development (NAFOSTED) under grant 103.02.2012.51.

\section{REFERENCES}

[1] Q.S. Mei, K. Lu, Prog. Mater. Sci. 52 (2007) 1175

[2] M. Takagi, J. Phys. Soc. Jpn. 9 (1954) 359

[3] T. Ohashi, K. Kuroda, H. Saka, Philos. Mag. B 65 (1992) 1041

[4] K. Sasaki, H. Saka, Philos. Mag. A, 63 (1991) 1207

[5] Q. Jiang, H.X. Shi, M. Zhao, J. Chem. Phys. 111 (1999) 2176

[6] A.N. Goldstein, C.M. Ether, A.P. Alivisatos, Science 256 (1992) 1425

[7] L. T Canham, Appl. Phys. Lett., 57 (1990) 1046

[8] P. Pawlow, Z. Phys. Chem. Stoechiom, Verwandtschaftsl 65 (1909) 545

[9] K. K. Nanda, S. N. Sahu, S. N. Behera, Phys. Rev. A 66 (2002) 13208

[10] Q. Jiang, S. Zhang, M. Zhao, Mater. Chem. Phys. 82 (2003) 225

[11] P. N. Keating, Phys. Rev. 145 (1966) 637

[12] R. Biswas, D.R. Hamann, Phys. Rev. Lett. 55 (1985) 2001

[13] S. Saito, S. Ohnishi, S. Sugano, Phys. Rev. B 33 (1986) 7036

[14] F. H. Stillinger,T.A. Weber, Phys. Rev. B, 31 (1985) 5262

[15] L. Pfeiffer, A. E. Gelman, K. A. Jackson, K. W. West, J. L. Batstone, Appl. Phys. Lett. 51 (1987) 1256

[16] S. R. Phillpot, J. F. Lutsko, D. Wolf, S. Yip, Phys. Rev. B 40 (1989) 2831

[17] S. R. Stiffler, M. O. Thompson, P. S. Peercy, Phys. Rev. B 43 (1991) 9851

[18] A. N. Goldstein, Appl. Phys. A, 62 (1996) 33

[19] C. P. Grigoropoulos, S. Moon, M. Lee, M. Hatano, K. Suzuki, Appl. Phys. A 69 (1999) S295

[20] S. Reber, W. Zimmermann, T. Kieliba, Sol. Energy Mater. Sol. Cells 65 (2001) 409

[21] R. Ishihara, F.C. Voogt, Solid State Phenom. 80 (2001) 163

[22] Y. W. Tang, J. Wang, X. C. Zeng, J. Chem. Phys., 124 (2006) 236103

[23] V. S. Dozhdikov, A. Y. Basharin, P. R. Levashov, J. Chem. Phys. 137 (2012) 054502

[24] Q. Zhang, Q. Li, M. Li, J. Chem. Phys., 138 (2013) 044504

[25] E. S. Zijlstra, A. Kalitsov, T. Zier, M.E. Garcia, Phys. Rev. X 3 (2013) 011005

[26] F. A. Lindemann, Phys. Z 11 (1910) 609

[27] A. Proykova, S. Pisov, R. Radev, P. Mihailov, I. Daykov, R. S. Berry, Vacuum 68 (2003) 87

[28] W. D. Kristensen, E. J. Jensen, R. M. J. Cotterill, J. Chem. Phys. 60 (1974) 4161

[29] C. L. Briant, J. J. Burton, J. Chem. Phys. 63 (1975) 2045

[30] R. D. Etters, J. B. Kaelberer, Phys. Rev. A 11 (1975) 1068

[31] J. B. Kaelberer, R. D. Etters, J. Chem. Phys. 66 (1977) 3233 
[32] R. D. Etters, J. B. Kaelberer, J. Chem. Phys. 66 (1977) 5112

[33] T. L. Beck, J. Jellinek, R. S. Berry, J. Chem. Phys. 87 (1987) 545

[34] S. J. Zhao, S. Q. Wang, D. Y. Cheng, H. Q. Ye, J. Phys. Chem. B 105 (2001) 12857

[35] G. Del Mistro, A. J. Stace, J. Chem. Phys. 98 (1993) 3905

[36] P. Shah, S. Roy, C. Chakravarty, J. Chem. Phys. 118 (2003) 10671

[37] T. Bachels, H. J. Gntherodt, R. Schfer, Phys. Rev. Lett. 85 (2000) 1250

[38] S. Plimpton, J. Comput. Phys. 117 (1995) 1

[39] H. Sakai, Surf. Sci., 351 (1996) 285

[40] T. Bachels, H.J. Guntherodt, Phys. Rev. Lett., 85 (2000) 1250

[41] Y. Qi, T. Cagin, W. L. Johnson, W. A. Goddard III, J. Chem. Phys., 115 (2001) 385

[42] S. Alavi, D.L. Thompson, J. Phys. Chem. A 110 (2006) 1518

[43] A. Kubo, Y. Wang, C.E. Runge, T. Uchida, B. Kiefer, N. Nishiyama, and T.S. Duffy, J. Phys. Chem. Solids 69 (2008) 2255

[44] V. V. Hoang, D. Ganguli, Physics Reports 518 (2012) 81

[45] D. K. Belashchenko, Russ. J. Phys. Chem. 80 (2006) 1968

[46] D. K. Belashchenko, E. A. Lobanov, G. F. Syrykh, Russ. J. Phys. Chem. 79 (2005) S135

[47] V. N. Novikov, E. Rossler, V. K. Malinovsky, N. V. Surovtsev, Europhys. Lett. 35 (1996) 289

[48] M. Forsblom, G. Grimvall, Phys. Rev.B 72 (2005) 054107

[49] V. V. Hoang, Philos. Mag. 91 (2011) 3443

[50] Z. H. Jin, P. Gumbsch, K. Lu, E. Ma, Phys. Rev. Lett. 87 (2001) 055703 